\title{
LOCALLY REFINABLE PARAMETRIC SNAKES
}

\author{
Anaïs Badoual, Daniel Schmitter and Michael Unser \\ Biomedical Imaging Group, École polytechnique fédérale de Lausanne (EPFL), Switzerland
}

\begin{abstract}
Shape segmentation is an active field of research in biomedical imaging. In this context, we present a new parameterization of a snake that is locally refinable. We introduce the possibility of locally increasing the approximation power of the parametric model by inserting basis functions at a specific location. This is controlled by a user-interface that permits the refinement of an initial segmentation around an anchor position selected by a user. Our approach relies on scaling functions that satisfy the refinement relation and are related to wavelets. We also derive explicit formulas for the energy functions associated to our new parameterization. We demonstrate the accuracy of our snake and its robustness under noisy conditions on phantom data. We also present segmentation results on real cell images, which are our main target. The algorithm is made freely available as a plugin for the open source platform Icy.
\end{abstract}

Index Terms - parametric snake, active contour, splines, segmentation, refinable, local refinement, scaling function.

\section{INTRODUCTION}

Parametric snakes [1] are widely used for user-interactive bioimage segmentation [2, 3, 4]. The shapes of biological structures often exhibit different levels of detail [5, 6]. For conventional snakes, this is [7] dealt with in a global fashion by simply increasing the number of control points resulting in an increase of the degrees of freedom of the model. While this improves the approximation power [8] of the snake model, it renders the optimization more challenging and increases the computation substantially. Moreover, introducing more degrees of freedom in the part of the curve where they are not required does not necessarily improve the segmentation outcome. On the other hand, few parameters allow for faster optimization $[9,10,11]$ but with less accurate approximation.

We propose a new parametric snake that has the ability to locally increase its approximation power. This allows for a more efficient allocation of the degrees of freedom of the snake by concentrating them on segments of higher complexity. For this purpose we exploit the refinability property of

\footnotetext{
This work was funded by the European Research Council under the European Union's Seventh Framework Programme (FP7/2007-2013) / ERC grant agreement 267439 and the Swiss National Science Foundation under Grant 200020-144355 .
}

scaling functions [12]. The primary contributions of this work are:

1. a novel parameterization of closed curves that are refinable locally;

2. a generic formulation of locally refinable snakes within that framework with the derivation of corresponding contour and region energies to speed up computations;

3. the demonstration of the benefits of refinability in the context of semi-interactive segmentation.

\section{LOCALLY REFINABLE PARAMETRIC CLOSED CURVES}

In this section we present our parameterization of locally refinable curves using refinable basis functions. For the reminder of the article, we focus on closed curves because such models have a wide range of applications in bioimaging, such as the segmentation of blobish, elliptical, or any other kind of closed structure $[5,13]$. However, the extension to open curves is straightforward. We first introduce the generic representation of closed curves and the refinement relation.

\subsection{Generic representation}

A 2D planar curve $\mathbf{r}$ can be described by a pair of one dimensional coordinate functions $(x(t), y(t))$ where $t \in \mathbb{R}$ is a continuous parameter. Each of these functions is parameterized by a suitable linear combination of shifted basis functions $\{\varphi(\cdot-k)\}_{k \in \mathbb{Z}}$ specified by a sequence of control points $\{\mathbf{c}[k]\}_{k \in \mathbb{Z}}$, such that

$$
\mathbf{r}(t)=\sum_{k \in \mathbb{Z}} \mathbf{c}[k] \varphi(t-k), t \in \mathbb{R} .
$$

We focus on closed (i.e. periodic) curves that are characterized by an $M$-periodic sequence of control points. We reexpress (1) as

$$
\mathbf{r}(t)=\sum_{k=0}^{M-1} \mathbf{c}[k] \varphi_{M}(t-k), t \in[0, M[,
$$

where $\varphi_{M}$ is the $M$-periodization of the basis function $\varphi$ defined by

$$
\varphi_{M}(t)=\sum_{k \in \mathbb{Z}} \varphi(t-k M) .
$$




\subsection{Scaling functions}

A valid scaling function $\varphi$ verifies the refinement relation given by

$$
\varphi(t)=\sum_{k \in \mathbb{Z}} h[k] \varphi(m t-k)
$$

where $m$ is called the refinement factor and $\{h[k]\}_{k \in \mathbb{Z}}$ are the coefficients of the filter associated to the refinement relation $[14,15]$. Thus, if $\varphi$ is a valid scaling function it can be expressed as a linear combination of its contracted version shifted by integers.

\subsection{Representation with local refinement}

We use basis functions that are valid scaling functions as given by (4) to parameterize closed curves. The refinement relation (4) is applied locally, i.e. only w.r.t. one particular control point $\mathbf{c}[p]$ associated to the shifted basis function $\varphi(t-p)$.

Proposition 1. A parametric curve that has been locally refined w.r.t. $\mathbf{c}[p]$ can be expressed as

$\mathbf{r}(t)=\sum_{\substack{k=0 \\ k \neq p}}^{M-1} \mathbf{c}[k] \varphi_{M}(t-k)+\sum_{l=l_{0}}^{l_{0}+N-1} \tilde{\mathbf{c}_{p}}[l] \varphi_{m M}(m t-m p-l)$,

where $N$ is the size of the discrete filter $h$, whose support is $\left[l_{0}, l_{0}+N\right]$ and $\tilde{\mathbf{c}_{p}}[k]=h[k] c[p]$.

Proof: Using (2) we write

$$
\mathbf{r}(t)=\sum_{\substack{k=0 \\ k \neq p}}^{M-1} \mathbf{c}[k] \varphi_{M}(t-k)+\mathbf{c}[p] \varphi_{M}(t-p)
$$

By (3) and (4), $\varphi_{M}(t-p)$ can be expressed as

$$
\begin{aligned}
\varphi_{M}(t-p) & =\sum_{k \in \mathbb{Z}} \varphi(t-p-k M) \\
& =\sum_{k \in \mathbb{Z}} \sum_{l \in \mathbb{Z}} h[l] \varphi(m t-m p-m k M-l) \\
& =\sum_{l \in \mathbb{Z}} h[l] \varphi_{m M}(m t-m p-l)
\end{aligned}
$$

Therefore,

$$
\begin{aligned}
\mathbf{c}[p] \varphi_{M}(t-p) & =\mathbf{c}[p] \sum_{l \in \mathbb{Z}} h[l] \varphi_{m M}(m t-m p-l) \\
& =\sum_{l \in \mathbb{Z}} \underbrace{\mathbf{c}[p] h[l]}_{\tilde{c_{p}}[l]} \varphi_{m M}(m t-m p-l) .
\end{aligned}
$$

By taking into account the size of the filter $h$, which is equal to $N$, as well as its localization on $\left[l_{0}, l_{0}+N\right]$ we can simplify the infinite sum in (8) to obtain

$$
\mathbf{c}[p] \varphi_{M}(t-p)=\sum_{l=l_{0}}^{l_{0}+N-1} \tilde{\mathbf{c}_{p}}[l] \varphi_{m M}(m t-m p-l) .
$$

By combining (9) and (6) we obtain (5).

The local refinement described by Proposition 1 allows the part of the curve initially controlled by $\mathbf{c}[p]$ to be described

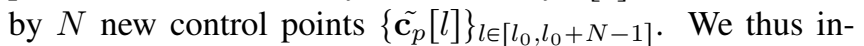
crease the approximation power of the curve at this specific location. This local refinability can be efficiently exploited for the construction of snakes as described in the next section. The local refinement of a parametric curve is illustrated in Fig. 1, where we have chosen $\varphi$ to be equal to the quadratic B-spline.

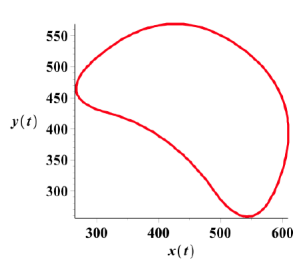

(a)

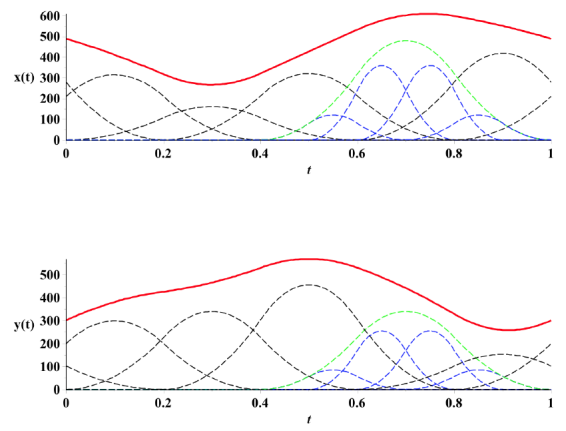

(b)
Fig. 1. Locally refinable curve. A parametric curve represented with the quadratic B-spline and $M=5$ (a) and its coordinate functions (b). The black dashed lines are the basis functions, the blue ones correspond to the refinement of the green one.

\section{LOCALLY REFINABLE SNAKE}

A snake is a deformable curve that is attracted towards the boundary of an object of interest [16]. It is guided by defining and minimizing a corresponding image-energy functional. It is generally composed by an edge-based energy computed from gradient information and a region-based energy that is capable of separating different homogeneous regions [17]. These two energies are combined as

$$
E_{\text {image }}=\alpha E_{\text {edge }}+(1-\alpha) E_{\text {region }},
$$

where $\alpha \in[0,1]$ is a trade-off parameter that balances the contribution of the two energies according to the application.

\subsection{Contour energy}

We consider a contour energy similar to the one proposed in [18] since it is independent of the parameterization. The edge- 
based energy is given by

$$
E_{\text {edge }}=-\oint_{\mathbf{r}} \mathbf{k}^{T}(\nabla f(\mathbf{r}) \times \mathrm{d} \mathbf{r}),
$$

where $\mathbf{k}=(0,0,1)$ is the outward vector orthonormal to the image plane, $\nabla f(x, y)$ is the within-plane gradient of the image $f$ at location $(x, y)$ on the curve, $\mathrm{d} \mathbf{r}$ denotes the tangent vector of the curve in the $3 \mathrm{D}$ space formed by the image plane and its orthogonal dimension and $\times$ is the $3 \mathrm{D}$ cross product.

Proposition 2. Let $\mathbf{r}$ be a locally refinable curve as described in Proposition 1. Then the edge-based energy term (11) can be expressed as

$$
\begin{aligned}
E_{\text {edge }}= & \sum_{\substack{k=0 \\
k \neq p}}^{M-1} c_{y}[k] \int_{0}^{M} G(\mathbf{r}(t)) \dot{\varphi}_{M}(t-k) \mathrm{d} t \\
& +m \sum_{l=l_{0}}^{l_{0}+N-1} \tilde{c}_{p_{y}}[l] \int_{0}^{M} G(\mathbf{r}(t)) \dot{\varphi}_{m M}(m t-m p-l) \mathrm{d} t,
\end{aligned}
$$

where $\dot{\varphi}(t)=\frac{\mathrm{d} \varphi(t)}{\mathrm{d} t}, G$ is given by

$$
G(x, y)=\int_{-\infty}^{x} g(\tau, y) \mathrm{d} \tau,
$$

$g=-\Delta f$, and $\Delta f$ is the Laplacian of the image $f$.

Proof: Using Green's theorem, (11) is expressed as the surface integral

$$
\begin{aligned}
E_{\text {edge }} & =-\iint_{\Omega} \Delta f(\mathbf{r}) \mathrm{d} x \mathrm{~d} y \\
& =\oint_{\mathbf{r}} G(\mathbf{r}) \mathrm{d} y=\int_{0}^{M} G(\mathbf{r}(t)) \frac{\mathrm{d} y(t)}{\mathrm{d} t} \mathrm{~d} t .
\end{aligned}
$$

Taking the derivative of (5) and combining it with (14), we obtain (12).

Equation (13) allows a faster implementation of the edgeenergy (c.f. Section 4).

\subsection{Region energy}

The region-based energy discriminates an object from its background by building a shell $\mathbf{r}_{\lambda}$ around the snake obtained by dilating $\mathbf{r}$ by a factor $\sqrt{2}$ with respect to its center of gravity. The contrast between the average intensity of the data enclosed by $\mathbf{r}$ and the data enclosed by the shell $\mathbf{r}_{\lambda}$ is minimized. This region energy is expressed as

$$
E_{\text {region }}=\frac{1}{|\Omega|}\left(\iint_{\Omega} f(\mathbf{r}) \mathrm{d} x \mathrm{~d} y-\iint_{\Omega_{\lambda} \backslash \Omega} f(\mathbf{r}) \mathrm{d} x \mathrm{~d} y\right),
$$

where $|\Omega|$ is the signed area enclosed by the contour.
Proposition 3. If $\mathbf{r}$ is locally refinable in the sense of Proposition 1, then the region-based energy (15) is given by

$$
\begin{aligned}
E_{\text {region }}= & \frac{1}{|\Omega|}\left(2 \sum_{\substack{k=0 \\
k \neq p}}^{M-1} c_{y}[k] \int_{0}^{M} F(\mathbf{r}(t)) \dot{\varphi}_{M}(t-k) \mathrm{d} t\right. \\
& +2 m \sum_{k=l_{0}}^{l_{0}+N-1}{\tilde{p_{y}}}_{\tilde{y}}[k] \int_{0}^{M} F(\mathbf{r}(t)) \dot{\varphi}_{m M}(m t-m p-k) \mathrm{d} t \\
& -\sum_{\substack{k=0 \\
k \neq p}}^{M-1} c_{y_{\lambda}}[k] \int_{0}^{M} F\left(\mathbf{r}_{\boldsymbol{\lambda}}(t)\right) \dot{\varphi}_{M}(t-k) \mathrm{d} t \\
& \left.-m \sum_{k=l_{0}}^{l_{0}+N-1} c_{p_{y_{\lambda}}}[k] \int_{0}^{M} F\left(\mathbf{r}_{\boldsymbol{\lambda}}(t)\right) \dot{\varphi}_{m M}(m t-m p-k) \mathrm{d} t\right),
\end{aligned}
$$

where

$$
F(x, y)=\int_{-\infty}^{x} f(\tau, y) \mathrm{d} \tau .
$$

The signed area enclosed by $\mathbf{r}$ is given by

$$
\begin{aligned}
|\Omega|= & -\sum_{\substack{k=0 \\
k \neq p}}^{M-1} \sum_{\substack{l=0 \\
l \neq p}}^{M-1} c_{y}[k] c_{x}[l] \int_{0}^{M} \varphi_{M}(t-k) \dot{\varphi}_{M}(t-l) \mathrm{d} t \\
& -m \sum_{\substack{k=0 \\
k \neq p}}^{M-1} \sum_{l=l_{0}}^{l_{0}+N-1} c_{y}[k]{\tilde{p_{x}}}_{p_{x}}[l] \int_{0}^{M} \varphi_{M}(t-k) \dot{\varphi}_{m M}(m t-m p-l) \mathrm{d} t \\
& -\sum_{k=l_{0}}^{l_{0}+N-1} \sum_{l=0}^{M-1} c_{p_{y}}[k] c_{x}[l] \int_{0}^{M} \varphi_{m M}(m t-m p-k) \dot{\varphi}_{M}(t-l) \mathrm{d} t \\
& -m \sum_{k=l_{0}}^{l_{0}+N-1} \sum_{l=l_{0}}^{l_{l_{0}+N-1}} c_{p_{y}}[k] \tilde{c}_{p_{x}}[l] \int_{0}^{M} \varphi_{m M}(m t-m p-k) \\
& \dot{\varphi}_{m M}(m t-m p-l) \mathrm{d} t .
\end{aligned}
$$

Proof: We rewrite (15) as

$$
\begin{aligned}
E_{\text {region }} & =\frac{1}{|\Omega|}\left(2 \iint_{\Omega} f(\mathbf{r}) \mathrm{d} x \mathrm{~d} y-\iint_{\Omega_{\lambda}} f\left(\mathbf{r}_{\lambda}\right) \mathrm{d} x \mathrm{~d} y\right) \\
& =\frac{1}{|\Omega|}\left(2 \oint_{\mathbf{r}} F(\mathbf{r}(t)) \frac{\mathrm{d} y(t)}{\mathrm{d} t} \mathrm{~d} t-\oint_{\mathbf{r}_{\lambda}} F\left(\mathbf{r}_{\lambda}(t)\right) \frac{\mathrm{d} y_{\lambda}(t)}{\mathrm{d} t} \mathrm{~d} t\right)
\end{aligned}
$$

The area can be expressed as $\Omega=\iint_{\Omega} \mathrm{d} x \mathrm{~d} y$, while Green's theorem yields the signed area

$$
|\Omega|=-\oint_{\mathbf{r}} y \mathrm{~d} x .
$$

Using equation (5) and its derivative and combining with (19) and (20), we obtain (16) and (18). 


\section{IMPLEMENTATION}

We implemented the proposed framework as a user-friendly open source plugin available for the bioimaging platform Icy [19]. The $\alpha$ parameter in (10) and the refinement factor $m$ can be adjusted by the user. We minimize $E_{\text {image }}$ using a Powelllike line search method where we explicitly use equations (12) and (16) to analytically compute the gradient of the energy w.r.t. the control points in order to accelerate the optimization process. To further speed up the computations, the images described by (13) and (17) are precomputed and stored in look-up tables. The plugin is freely available for Windows /Linux / Mac at http://bigwww.epfl.ch/algorithms.html (after acceptance of the publication).

\section{EXPERIMENTS AND VALIDATION}

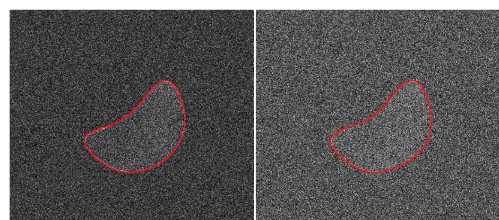

$\left(a_{1}\right)$

$\left(a_{2}\right)$

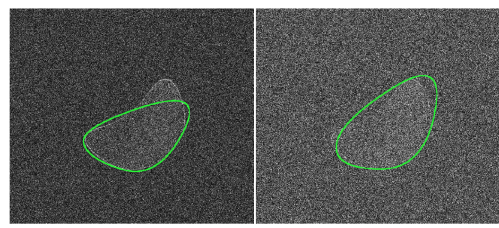

(a)

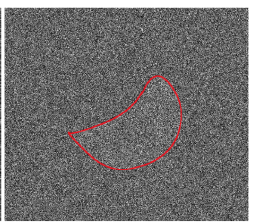

$\left(a_{3}\right)$

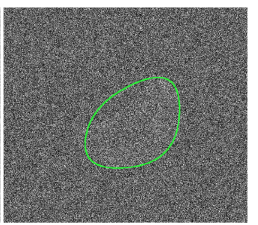

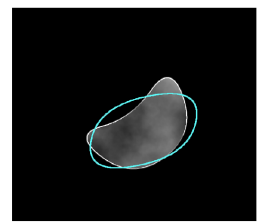

(b)

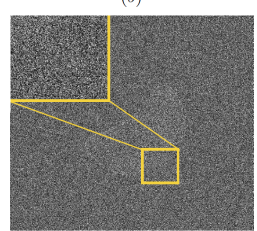

(c)
Fig. 2. Robustness w.r.t. noise of the locally refinable snake. (a) Comparison for different SNR between two quadratic Bspline snakes with $M=4$; locally refinable (top row) and traditional snake (bottom row). $\left(a_{1}\right) \mathrm{SNR}=-13.40 \mathrm{~dB} .\left(a_{2}\right)$ $\mathrm{SNR}=-16.16 \mathrm{~dB} .\left(a_{3}\right) \mathrm{SNR}=-17.35 \mathrm{~dB}$. (b) Initialization for both snakes. (c) Close-up of a boundary region between the test cell and its background, $\mathrm{SNR}=-17.35 \mathrm{~dB}$.

To show the accuracy and the rosbustness of our proposed method, we performed experiments on both phantom and real data. For this purpose, we used quadratic B-splines as scaling functions whose corresponding refinement filter is defined by its z-transform as $H(z)=\frac{1}{4}\left(1+z^{-1}\right)^{3}$. The refinement factor corresponds to $m=2$.

\subsection{Phantom data}

A test image simulating fluorescence microscopy showing a cell was created. The image was then corrupted by different levels of additive Gaussian white noise. For each image, we segmented the structure of interest using both our locally refinable and a traditional non-refinable parametric snake [17] using the same initialization and $M=4$ control points. A first optimization of the snake is performed without local refinement. Then, the user clicks on a desired control point and the corresponding basis function is refined. The optimization is launched again. SNRs corresponding to the noise level and Jaccard indices at both optimization steps are shown in Table 1 and illustrated in Fig. 2. Both Table 1 and Fig. 2 show the improved accuracy induced by the local refinement. It also illustrates that the scheme is robust to noise.

Table 1. Jaccard indices for segmentation of (noisy) data.

\begin{tabular}{ccc}
\hline \hline SNR $[\mathrm{dB}]$ & with local refinement & without local refinement \\
\hline-7.44 & 0.95 & 0.79 (fail) \\
-10.95 & 0.95 & 0.73 (fail) \\
-13.40 & 0.95 & 0.79 (fail) \\
-15.07 & 0.94 & 0.81 (fail) \\
-16.16 & 0.94 & 0.83 (fail) \\
-16.88 & 0.92 & 0.84 (fail) \\
-17.35 & 0.91 & 0.78 (fail) \\
\hline \hline
\end{tabular}

\subsection{Real data}

We have applied our snake to real fluorescence microscopy images and obtained promising results. Fig. 3 shows the segmentation of a sickle cell obtained with a locally refinable parametric snake on such an image.

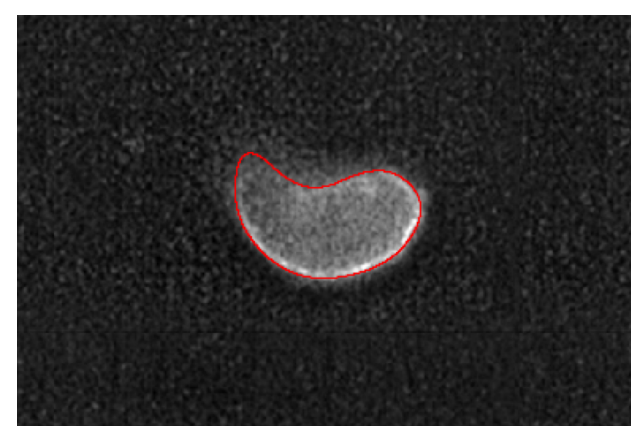

Fig. 3. Segmentation of a sickle cell on a fluorescence microscopy image using the locally refinable parametric snake.

\section{CONCLUSIONS}

We have presented a new and complete formulation of a locally refinable parametric snake for image segmentation. We provide explicit expressions for the refined parametric curve as well as the corresponding energy function. Our method is generic and can be used with any valid scaling function. We have demonstrated its ability of improving segmentation results as well as its robustness under noisy conditions. The corresponding software is available as an open source and user-interactive plugin. 


\section{REFERENCES}

[1] T.F. Chan and L.A. Vese, "Active contours without edges," IEEE Transactions on Image Processing, vol. 10, no. 2, pp. 266-277, February 2001.

[2] J. Tang and S.T. Acton, "Vessel boundary tracking for intravital microscopy via multiscale gradient vector flow snakes," IEEE Transactions on Biomedical Engineering, vol. 51, no. 2, pp. 316-324, February 2004.

[3] A. Dufour, R. Thibeaux, E. Labruyere, N. Guillen, and J.-C. Olivo-Marin, "3-D Active meshes: Fast discrete deformable models for cell tracking in 3-D time-lapse microscopy," IEEE Transactions on Image Processing, vol. 20, no. 7, pp. 1925-1937, July 2011.

[4] M.A.T. Figueiredo, J.M.N. Leitão, and A.K. Jain, "Unsupervised contour representation and estimation using B-splines and a minimum description length criterion," IEEE Transactions on Image Processing, vol. 9, no. 6, pp. 1075-1087, June 2000.

[5] E. Meijering, "Cell segmentation: 50 years down the road," IEEE Signal Processing Magazine, vol. 29, no. 5, pp. 140-145, September 2012.

[6] B. Li and S.T. Acton, "Active contour external force using vector field convolution for image segmentation.," IEEE Transactions on Image Processing, vol. 16, no. 8, pp. 2096-2106, August 2007.

[7] P. Brigger, J. Hoeg, and M. Unser, "B-Spline snakes: A flexible tool for parametric contour detection," IEEE Transactions on Image Processing, vol. 9, no. 9, pp. 1484-1496, September 2000.

[8] M. Jacob, T. Blu, and M. Unser, "Sampling of periodic signals: A quantitative error analysis," IEEE Transactions on Signal Processing, vol. 50, no. 5, pp. 11531159, May 2002.

[9] F. Precioso and M. Barlaud, "B-spline active contour with handling of topology changes for fast video segmentation.," EURASIP Journal on Applied Signal Processing, vol. 2002, no. 6, pp. 555-560, January 2002.

[10] Y. Zhang, B.J. Matuszewski, A. Histace, and F. Precioso, "Statistical model of shape moments with active contour evolution for shape detection and segmentation.," Journal of Mathematical Imaging and Vision, vol. 47, no. 1-2, pp. 35-47, 2013.

[11] G. Aubert, M. Barlaud, O.D. Faugeras, and S. JehanBesson, "Image segmentation using active contours: Calculus of variations or shape gradients?," SIAM Journal of Applied Mathematics, vol. 63, no. 6, pp. 21282154, 2003.
[12] Martin Vetterli and Jelena Kovačevic, Wavelets and Subband Coding, Prentice-Hall, Inc., Upper Saddle River, NJ, USA, 1995.

[13] O. Dzyubachyk, J. Van Cappellen, W. A.and Essers, W. J. Niessen, and E. Meijering, "Advanced levelset-based cell tracking in time-lapse fluorescence microscopy," IEEE Transactions on Medical Imaging, vol. 90, no. 3, pp. 852-867, March 2010.

[14] S.G. Chang, Z Cvetković , and M. Vetterli, "Locally adaptive wavelet-based image interpolation.," IEEE Transactions on Image Processing, vol. 15, no. 6, pp. 1471-1485, June 2006.

[15] M. Antonini, M. Barlaud, P. Mathieu, and I. Daubechies, "Image coding using wavelet transform," IEEE Transactions on Image Processing, vol. 1, no. 2, pp. 205-220, april 1992.

[16] X. Bresson, S. Esedoglu, P. Vandergheynst, J.-P. Thiran, and S. Osher, "Fast global minimization of the active contour/snake model," Journal of Mathematical Imaging and Vision, vol. 28, no. 2, pp. 151-167, 2007.

[17] R. Delgado-Gonzalo, P. Thévenaz, C.S. Seelamantula, and M. Unser, "Snakes with an ellipse-reproducing property," IEEE Transactions on Image Processing, vol. 21, no. 3, pp. 1258-1271, March 2012.

[18] M. Jacob, T. Blu, and M. Unser, "Efficient energies and algorithms for parametric snakes," IEEE Transactions on Image Processing, vol. 13, no. 9, pp. 1231-1244, September 2004.

[19] F. de Chaumont, S. Dallongeville, N. Chenouard, N. Hervé, S. Pop, T. Provoost, V. Meas-Yedid, P. Pankajakshan, T. Lecomte, Y. Le Montagner, T. Lagache, A. Dufour, and J.-C. Olivo-Marin, "Icy: An open bioimage informatics platform for extended reproducible research," Nature Methods, vol. 9, no. 7, pp. 690-696, July 2012. 